\title{
Acknowledgement to reviewers (2015)
}

\author{
Philippe Garrigues ${ }^{1}$
}

Received: 7 April 2016/Accepted: 17 April 2016/Published online: 19 May 2016

(C) Springer-Verlag Berlin Heidelberg 2016

Philippe Garrigues, Editor-in-chief of Environmental Science and Pollution Research (ESPR) Journal, and the whole editorial team would like to express their gratitude to all the reviewers who contributed through 2015 to the journal's dynamic.

We thank our generous reviewers for sharing their expertise, their time and professionalism that helped maintain the journal's quality. Your work is the key to our success.

First, we wish to thank the 25 most effective and responsive reviewers in 2015 in the following list:

Tsang Daniel, Hong Kong

Luo Chunling, China

Ok Yong Sik, South Korea

Hecker Markus, Canada

Zhou Xiaoqi, Australia

Cameselle Claudio, Spain

Jiang Yumei, China

Nakano Takeshi, Japan

Besis Athanasios, Greece

Wang Jun, China

Huang Wenjuan, China

Wei Binnian, USA

Katsoyiannis Athanasios, Belgium

Cao Jiashun, China

Kabiersch Grit, Finland

Sarakha Mohamed, France

Binh Minh Tu, Vietnam

Cao Wenbin, China

Liu Enfeng, China

Codling Garry, UK

Huang Qifei, China

Kim Jongmin, USA

Liu Weitao, China

Wei Chaoyang, China

Responsible editor: Philippe Garrigues

Electronic supplementary material The online version of this article

(doi:10.1007/s11356-016-6705-2) contains supplementary material,

which is available to authorized users.

Philippe Garrigues

p.garrigues@ism.u-bordeaux1.fr

1 University of Bordeaux, Talence Cedex, France 